\title{
Expression of PD-LI in cervical carcinoma and its impact on survival associated with T-cell infiltration and FoxP3 expression
}

This article was published in the following Dove Press journal: Cancer Management and Research

\author{
Rafael M Grochot ${ }^{1,2}$ \\ Janaína Brollo ${ }^{2}$ \\ Floriano Riva Neto ${ }^{2,3}$ \\ Aline C Tregnago ${ }^{2,3}$ \\ Cassiano Scholze ${ }^{3}$ \\ Rui Norris ${ }^{3}$ \\ Sargeele Silva ${ }^{2}$ \\ Débora $C$ Weschenfelder ${ }^{2}$ \\ André B Reiriz ${ }^{1,2}$ \\ Lessandra Michelin' \\ Fábio F Pasqualotto' \\ 'Department of Health Sciences, School \\ of Medicine, University of Caxias do Sul, \\ Caxias do Sul, RS, Brazil; ' 2 UNACON \\ Cancer Center, Caxias do Sul General \\ Hospital, Caxias do Sul, RS, Brazil; ${ }^{3}$ CPM \\ Laboratory, Caxias do Sul, RS, Brazil
}

Correspondence: Rafael M Grochot Caxias do Sul General Hospital,

University of Caxias do Sul, Rua Professor Antônio Vignoli, 255 - Bairro Petrópolis,

Caxias do Sul, RS 95070-56I, Brazil

Tel +55 5432187200

Email rmgrocho@ucs.br
Background: The PD-1/PD-L1 signaling axis is currently the most elucidated mechanism for tumor evasion of T-cell-mediated immunity. Nevertheless, few data are available regarding its impact on cervical cancer and the relationship with lymphocytic infiltrates.

Methods: A retrospective assessment of all cases of cervical neoplasia treated in Caxias do Sul General Hospital, Brazil, between 2012 and 2016 was performed. Clinical and pathological data were collected from electronic records and analyzed. Original slides were independently reviewed by three pathologists to confirm diagnoses and to assess the immunohistochemical expression of PD-L1 and FoxP3 in tumor cells and lymphocytic infiltrates.

Results: PD-L1 staining was present in $32.2 \%$ of the 59 cervical samples. Median overall survival time of the PD-L1-negative group was 47.8 months, a time point not yet reached by the PD-L1-positive group ( $p=0.968$ ). Median progression-free survival was 24.3 months for PD-L1-negative and 11.5 months for PD-L1-positive patients $(p=0.263)$. PD-L1 staining was found in $27.1 \%$ of the lymphocytic infiltrates, and survival analysis revealed no difference between PD-L1-positive and PD-L1-negative samples. There was no impact on survival related to FoxP3 staining in neither tumor samples nor lymphocytic infiltrates.

Conclusion: Although the median progression-free survival times differed, the difference was not statistically significant. Our study corroborates the rationale that PD-L1 expression in cervical neoplasms has no impact on survival. PD-L1 expression in peritumoral lymphocytes revealed no impact on infiltration volume nor survival.

Keywords: uterine cervical neoplasms, tumor-infiltrating lymphocytes, cancer, tumor microenvironment, survival

\section{Introduction}

Cervical cancer is the fourth most frequent cancer in woman worldwide with an estimated 570,000 new cases in 2018, representing 6.6\% of all female cancers. ${ }^{1}$ Most cases are squamous cell carcinoma followed by adenocarcinomas. Approximately $90 \%$ of deaths from cervical cancer occur in low- and middle-income countries. In Brazil, cervical cancer is the $2^{\text {nd }}$ most common cancer in women aged $15-44$ years and about 16,000 new cervical cancer cases are diagnosed annually. ${ }^{2}$

Human papillomavirus (HPV) infection is a well-established cause of cervical cancer. $^{3-5}$ The immune system plays an important role in HPV infections under conditions of high immunogenic reactivity. ${ }^{6}$ Immunosuppressed women have an increased incidence of HPV infection, cervical intraepithelial neoplasia (CIN) and progression to invasive neoplasia. $^{7-9}$ 
The programmed cell death protein-1/programmed death-ligand 1 (PD-1/PD-L1) signaling axis is currently the most elucidated mechanism for tumor evasion of $\mathrm{T}$ cell-mediated immunity. PD-1/PD-L1 antibody blockade has been widely proven to be effective against several cancers. Retrospective analyses have successfully associated PD-L1 expression to oncologic prognosis regardless of therapeutic strategy in melanoma, ovarian and kidney cancer. ${ }^{10,11}$ However, few data are available regarding its expression in cervical cancer. ${ }^{12}$

Recently, tumor infiltration by regulatory $\mathrm{T}$ lymphocytes (Treg) positive for forkhead box protein P3 (FoxP3) expression has been investigated as a potential prognostic immune biomarker. Nevertheless, discrepant results have been reported and its relationship with the PD-1/PD-L1 axis remains unclear. ${ }^{13,14}$

We assessed the impact of PD-L1 and FoxP3 expression in cervical tumor cells and intratumoral lymphocytes on survival outcomes.

\section{Methods}

A search for cases of cervical cancer in Caxias do Sul General Hospital database covering 2012 through 2016 was performed. Clinical and pathological data collected are summarized in Table 1. Study endpoints were date of diagnosis, progression-free survival (PFS) and overall survival (OS).

The original hematoxylin-eosin (HE) slides were reviewed for diagnosis confirmation. Immunohistochemistry (IHC) slides were independently scored by 3 pathologists. Discordant cases were reviewed and consensus was achieved for all cases.

Briefly, IHC was carried out using 3- $\mu$ m-thick sections obtained from formalin-fixed paraffin-embedded tissue. The slides were deparaffinized and rehydrated. Antigen retrieval was performed in Tris-EDTA (Thermo Fischer, Waltham, MA) at $95^{\circ} \mathrm{C}$ for 20 mins. The slides were incubated with primary antibodies PD-L1 (1:50 dilution, RTB-PDL1, Bio SB ${ }^{\circledR}$ ) and FoxP3 (1:100 dilution, EP340, Cell Marque ${ }^{\circledR}$ ) overnight. These antibodies were marked with dark brown chromogen (DAB) to allow its observation in light microscopy. Human placenta (trophoblasts) was used as positive control.

PD-L1 and FoxP3 expression levels were assessed based on percentage and intensity of staining. Staining for PD-L1 was considered positive in a membrane pattern only and FoxP3 was considered positive in nuclear pattern only. Other staining patterns were disregarded.
Table I Clinical and pathological data

\begin{tabular}{|l|l|l|}
\hline \multicolumn{2}{|l|}{ Age at diagnosis (median) } & 44 years \\
\hline Smoking history & Yes & $28.8 \%(n=17)$ \\
& No & $71.2 \%(n=42)$ \\
\hline Histologic subtype & Squamous cell & $86.4 \%(n=5 I)$ \\
& Adenocarcinoma & $13.6 \%(n=8)$ \\
\hline PS & 0 & $28.8 \%(n=17)$ \\
& I & $62.7 \%(n=32)$ \\
& 2 & $5.1 \%(n=3)$ \\
& 3 & $3.4 \%(n=2)$ \\
& 4 & - \\
\hline CS (FIGO, 2009) & I & $10.1 \%(n=6)$ \\
& II & $30.5 \%(n=18)$ \\
& III & $40.7 \%(n=24)$ \\
& IVA & $13.6 \%(n=8)$ \\
& IVB & $5.1 \%(n=3)$ \\
\hline Treatament & Surgery (frontline) & $27.1 \%(n=16)$ \\
& CT-RT & $64 \%(n=38)$ \\
& Brachytherapy & $32.2 \%(n=19)$ \\
& RT (exclusive) & $16 \%(n=9)$ \\
\hline Endpoint & Progression & $47.5 \%(n=28)$ \\
& Death & $33.9 \%(n=20)$ \\
& Follow-up (median) & 26.2 months \\
\hline
\end{tabular}

Abbreviations: PS, performance status; CS, clinical stage.

Intratumoral lymphocytes were quantified, and infiltration was classified into weak, moderate or strong categories.

Absolute and relative frequencies were used for the descriptive statistical analysis of variables. Fisher's exact test and a two-tailed $\chi^{2}$ test were performed when applicable. Survival rates were calculated with the KaplanMeier method and analyzed with the log-rank test. Univariate Cox proportional models were used to determine hazard ratios (HRs), which represent the risk of death or progression among the different groups. All statistical analyses were conducted with the Statistical Package for the Social Sciences (SPSS) version 22.0.

All researchers signed a secrecy and confidentiality agreement after receiving approval by the Institutional Scientific Council and by the National Research Ethics Committee. Our study of biomarkers was conducted according to REMARK recommendations. ${ }^{15}$

\section{Results}

We found 155 patients diagnosed with cervical cancer in our institution. Twenty-five patients were excluded due to diagnostic discrepancies, and 57 patients had no paraffin 
tissue blocks available. After revision of original HE slides, 13 patients were excluded due to insufficient material for analysis, and one patient was excluded due to diagnostic nonconformity. The flowchart of the studied cohort is shown in Figure 1.

The median age of the patients was 44 years, and $28.8 \%$ had a history of smoking. Squamous cell carcinoma was the most commonly diagnosed histological type, accounting for $86.4 \%(n=51)$ of the cases, followed by adenocarcinoma $(n=8)$. At the time of diagnosis, $28.8 \%$ $(n=17)$ of the patients exhibited Performance status (PS) score of 0 and $62.7 \%(n=32)$ PS 1 ; three patients exhibited PS 2, and two PS 3. Six (10.1\%) patients presented with Clinical stage (CS) I, 18 (30.5\%) CS II, 24 (40.7\%) CS III, eight (13.6\%) CS IVA and three (5.1\%) CS IVB.

Primary surgical treatment was provided to $27.1 \%$ of the patients $(n=16)$, whereas $64 \%(n=38)$ received definitive chemoradiotherapy; $19(32.2 \%)$ patients underwent complementary brachytherapy. Nine patients (16\%) received radiotherapy exclusively. The median follow-up was 26.2 months. Disease progression was objectively documented in $47.5 \% \quad(n=28)$ of the cases. Twenty $(33.9 \%)$ deaths occurred in the cohort during the followup period.

\section{Cervical tumoral tissue assessment}

Of all the evaluated clinical and pathological variables (age, smoking, PS, histological subtype, CS, treatment modality and response outcome), none alone had an impact on the variability of PD-L1 expression among the studied neoplastic specimens. PD-L1 expression was observed in $32.2 \%(n=19)$ of the cervical samples, five of which $(8.5 \%)$ exhibited PD-L1 expression above 50\% (Figure 2). The median OS time of the PD-L1-negative group was 47.8 months, a time point not reached yet by the PD-L1-positive group ( $p=0.968$; Figure 3 ). The median progression-free survival was 24.3 months for the PD-L1negative group and 11.5 months for the PD-L1-positive group ( $p=0.263$; Figure 4 ).

Immunohistochemistry for FoxP3 revealed upregulation in $64.4 \%(n=38)$ of the tumor specimens; however, tumoral FoxP3 expression had no impact on survival outcomes (data not shown). Analysis of the PD-L1/FoxP3 ratio revealed that $57.9 \%(n=11)$ of $P D-L 1$-positive tumors were positive for FoxP3 versus $67.5 \%(n=27)$ of the PDL1-negative specimens $(p=0.56)$.

\section{Peritumoral lymphocytic infiltrate assessment}

Weak peritumoral lymphocytic infiltration was found in $57.6 \%(n=34)$ of the samples, moderate infiltration in $23.7 \%(n=14)$ and strong infiltration in $18.6 \%(n=11)$. Stratification by lymphocytic infiltrate volume showed no significant association with survival outcomes (Figure 5). Positive PD-L1 staining was observed in $27.1 \%(n=16)$ of lymphocytic infiltrates, and survival analysis showed no difference between positive and negative infiltrates.

A total of $93.2 \% \quad(n=55)$ of lymphocytic infiltrates expressed FoxP3, with no impact on survival outcomes (data not shown). Furthermore, 94.7\% of PD-L1-positive tumors exhibited positive staining for FoxP3 in their respective peritumoral infiltrates versus $92.5 \%(n=40)$ of

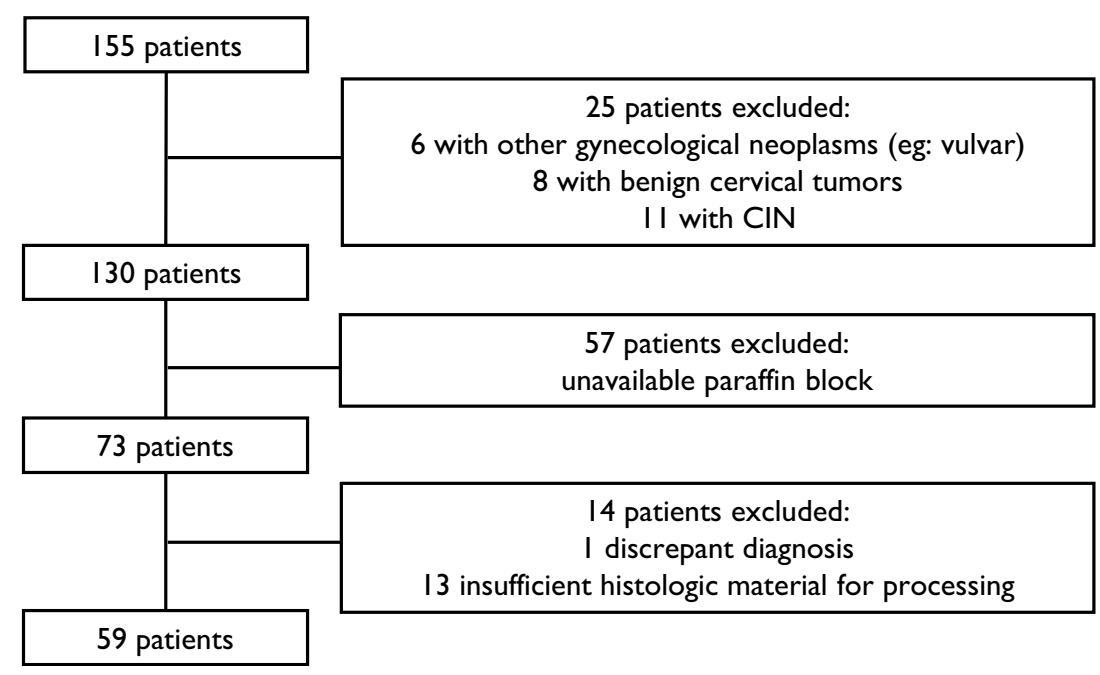

Figure I Flowchart of cohort recruitment. 


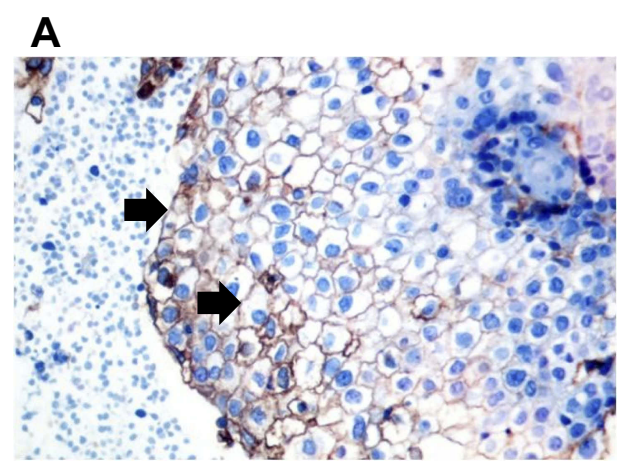

\section{B}

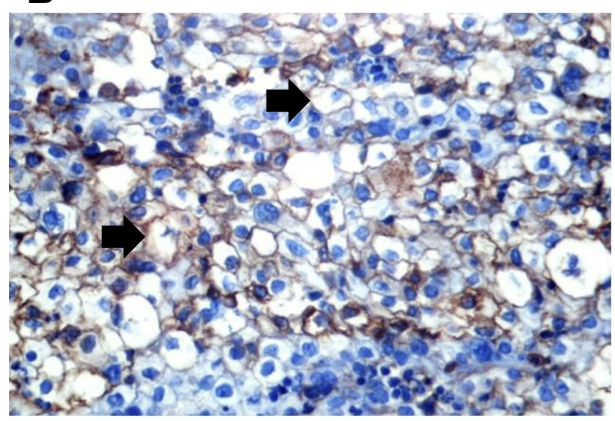

C

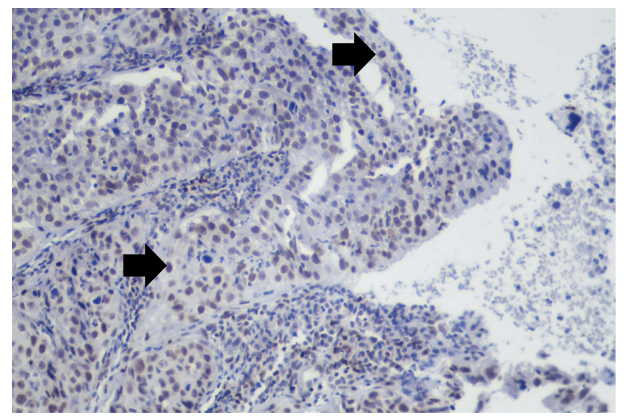

D

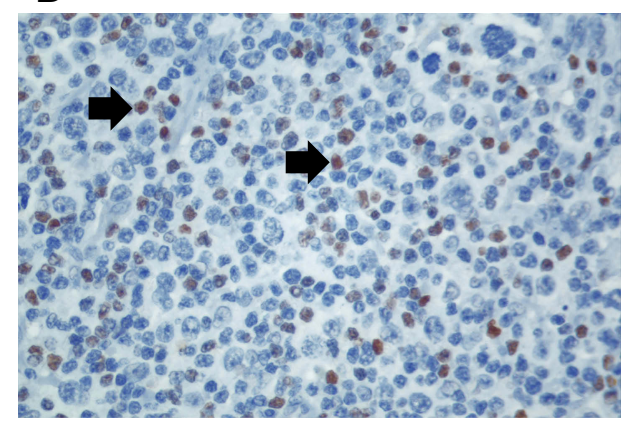

Figure 2 Immunohistochemical staining of PD-LI was considered positive if cell membrane staining was detected, and FoxP3 staining was considered positive if nuclear staining was detected. Black arrows point to staining detection areas. PD-LI-positive squamous cell cervical neoplasia (A); 400× magnification. PD-LI-positive peritumoral inflammatory infiltrate (B); 400x magnification. FoxP3-positive squamous cell cervical neoplasia (C); 200x magnification. FoxP3-positive peritumoral inflammatory infiltrate (D; 400x magnification).

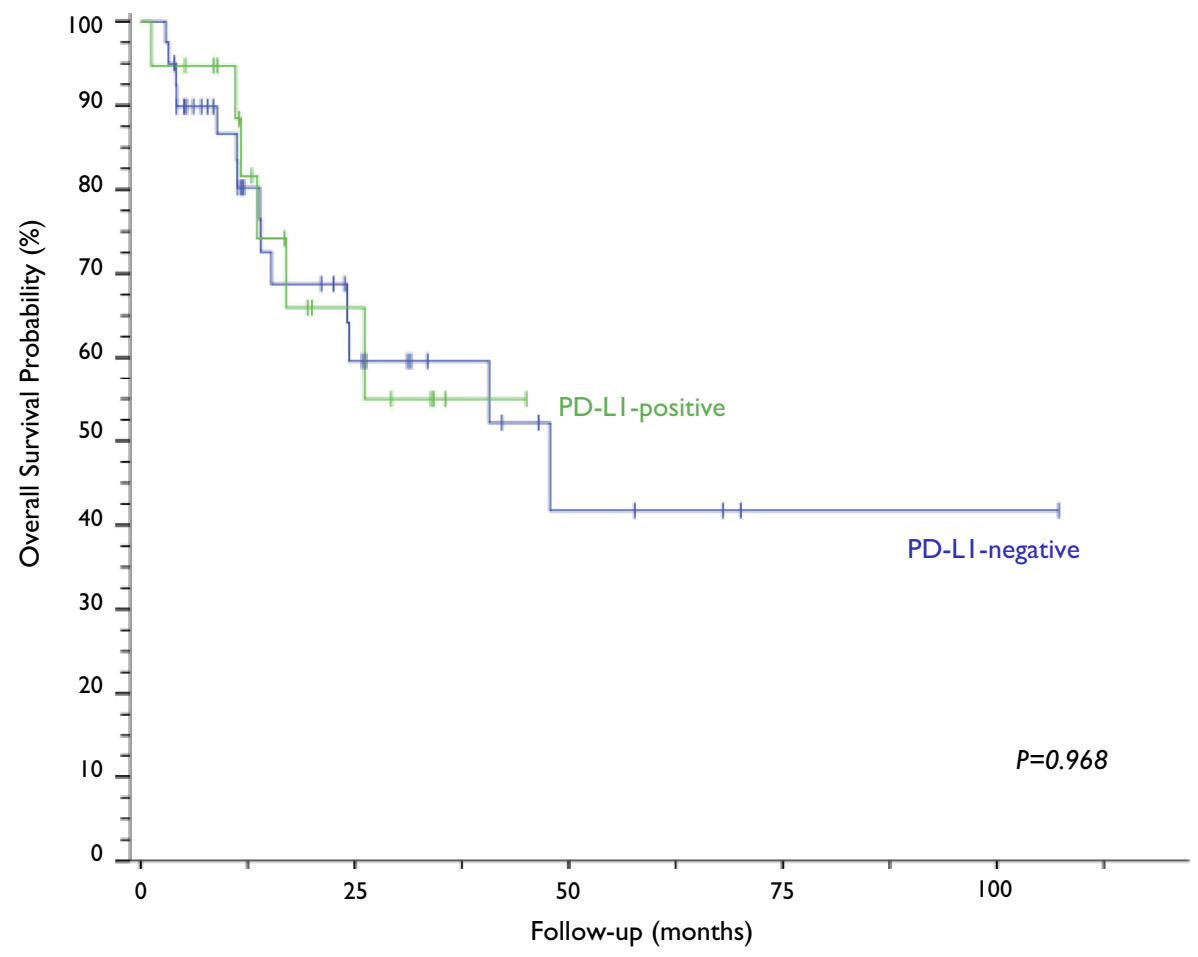

Figure 3 Kaplan-Meier curves and log-rank test analysis of the overall survival of patients with cervical neoplasia based on PD-LI expression. 


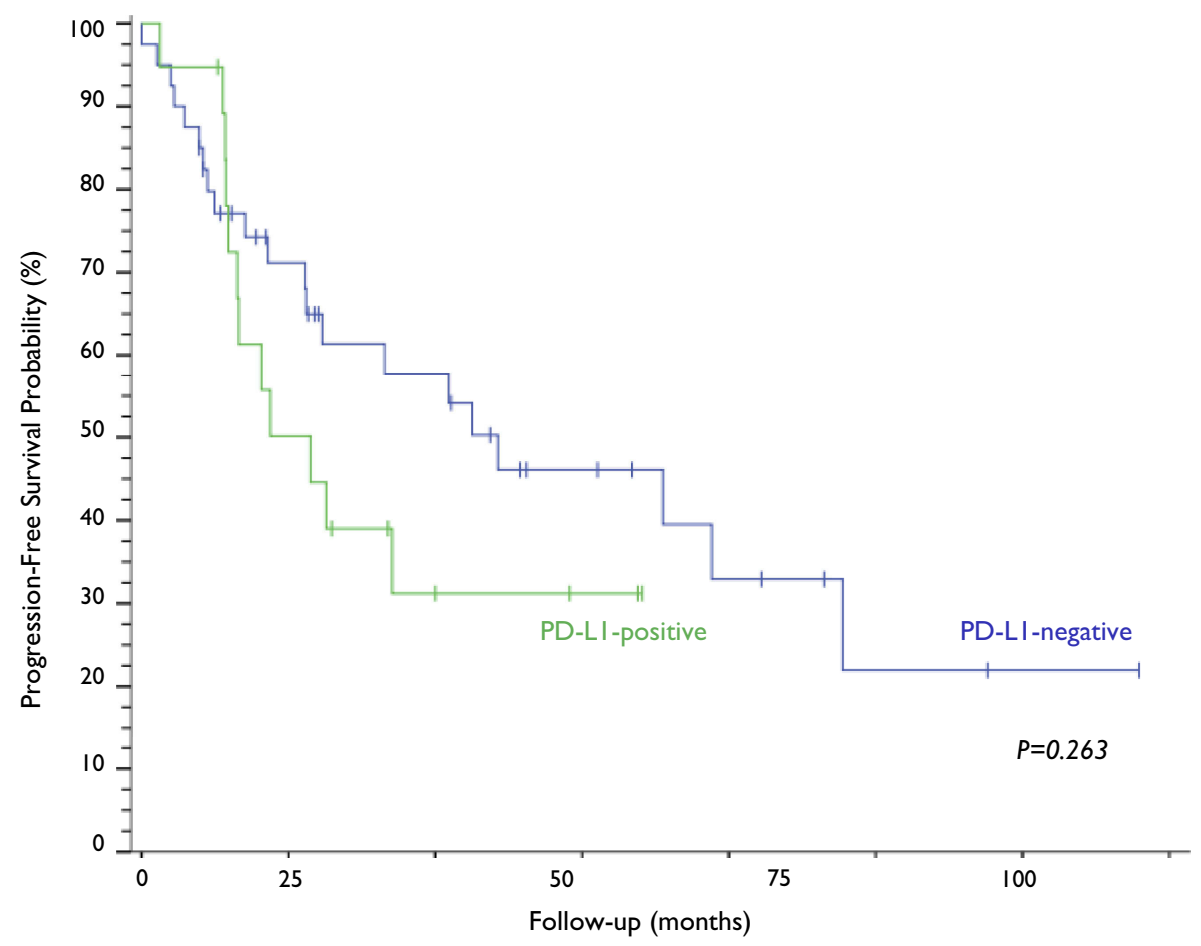

Figure 4 Kaplan-Meier curves and log-rank test analysis of the progression-free survival of patients with cervical neoplasia based on PD-LI expression.
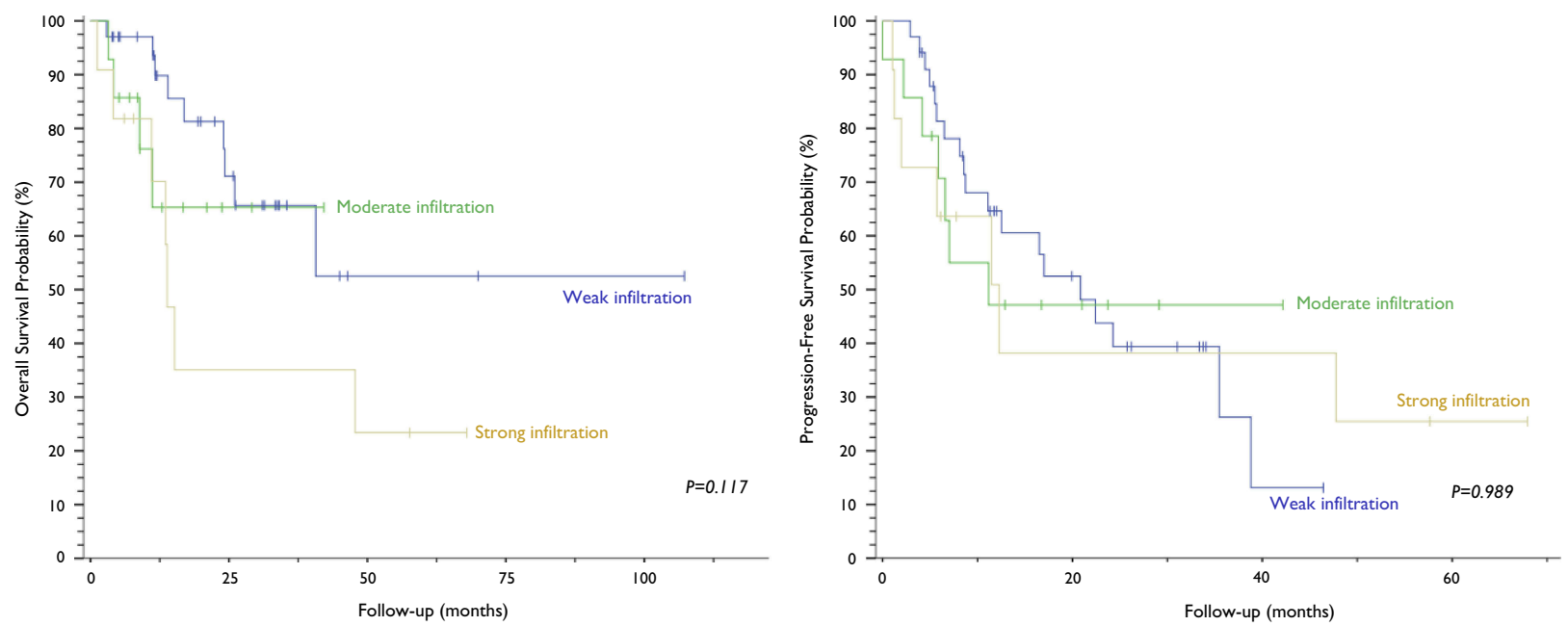

Figure 5 Kaplan-Meier curves and log-rank test analysis of overall survival (left) and progression-free survival (right) of patients with cervical neoplasia based on quantification of peritumoral lymphocytic infiltration.

infiltrates associated with PD-L1-negative cervical neoplasms $(p>0.99)$.

\section{Discussion}

\section{Cervical tumoral tissue assessment}

PD-L1 expression in cervical tumor cells has been previously evaluated; nonetheless, conflicting results have been reported regarding its impact on survival outcomes.
In 2017, Enwere et al assessed tumoral PD-L1 expression in patients with locally advanced cervical cancer. Their data regarding prevalence of PD-L1 expression are intriguing: $87.9 \%$ of their samples were considered positive at a cutoff point of $\geq 1 \%$ and $39.7 \%$ at $\geq 50 \%{ }^{12}$ Applying a cutoff point of $\geq 50 \%$, PD-L1 exhibited positive staining in only $8.5 \%$ of our samples. There was no association between PD-L1 expression and clinicopathological characteristics - age, CS, tumor size and lymph node 
status. Similarly, clinical and pathological variables of the present study had no impact on prognosis or on PD-L1 tumoral expression. In agreement with our results and with the findings of Heeren et al, ${ }^{16}$ their study also failed to detect an association between survival and tumoral expression of this biomarker. Karim et $\mathrm{al}^{17}$ found similar results. Only patients in CSs IB-II were included in their analysis and the authors suggest that PD-L1 expression can be different at more advanced disease stages. Nevertheless, PD-L1 expression was found in $19 \%$ of their samples and in $32.2 \%(n=19)$ of our specimens. Our study included patients at CSs III and IV, and subgroup analysis still also failed to show an impact on the expression of this biomarker and on survival outcomes.

We noted a distinct trend towards shorter progressionfree survival in patients expressing PD-L1 - 11.5 months versus 24.3 months for PD-L1 negative patients. Yet, no statistical significance was met $(p=0.263)$. Overall, by itself, IHC detection of tumoral PD-L1 does not seem to be an adequate biomarker for prediction of survival in cervical cancer.

Recently, the expression of FoxP3 has been examined in non-hematopoietic cells - including cancer cells; however, variable relationship between its tumoral expression and prognosis has been reported. In concordance with Huang et al, we found high levels of FoxP3 expression in tumoral cervical cells. ${ }^{18}$ While no impact on survival was met, previous studies observed that FoxP3 expression was positively related to the invasiveness of cervical lesions. ${ }^{19}$ These findings support the hypothesis that FoxP3 upregulation by tumor cancer cells might endow them with the immunosuppressive power of Treg, facilitating an escape from immune surveillance by downregulating T-cell function. ${ }^{20}$

\section{Peritumoral lymphocytic infiltrate assessment}

As previously stated, PD-L1 tumoral expression alone has failed to be associated with survival outcomes in cervical cancer. Conversely, Wang et al found that it seems to have a predictive value when combined with other immune features, such as the CD8+/CD4+CD25+FoxP3+ T lymphocyte ratio. $^{21}$

Evidence suggests that neoplasms associated with HPV exhibit a lack of an HPV-specific response by cytotoxic and type 1 helper T (Th1) lymphocytes. Also, scarce intratumoral infiltrates of CD8+ lymphocytes and abundant Treg seem to represent an independent prognostic factor for OS. ${ }^{9,16-25,26}$ Matsumoto et al examined intratumoral neutrophil infiltration $(\mathrm{CD} 66 \mathrm{~b}+$ cells) in 250 cervical cancer specimens. Recurrence rate was significantly associated with density of neutrophilic infiltrates. Five-year OS was $77.5 \%$ for the "TAN-low" group (low-density infiltration), $58.7 \%$ for the "TAN-med" group (moderate infiltration) and $17 \%$ for the "TAN-high" group (high-density infiltration). Progression-free survival was estimated at 73.8\%, 50.7\% and $11.3 \%$, respectively, over the same period. ${ }^{27}$ Applying the same stratification for lymphocytic infiltration, we found no impact on survival.

Regulatory $\mathrm{T}$ cells FoxP3+ are a class of immunesuppressive lymphocytes involved in tumoral immune evasion. ${ }^{18}$ Shang et al conducted a meta-analysis to assess the prognostic effect of Treg FoxP3+ infiltration across different cancers. The overall pooled analysis found an unfavorable prognostic effect, and this association was unanimous and particularly strong among studies of cervical cancer. ${ }^{14}$ In fact, $93.2 \%(n=55)$ of our lymphocytic infiltrates revealed FoxP3+, possibly corroborating the rationale that Treg might consist of an escape mechanism of cervical neoplasms to immune response.

The PD-1/PD-L1 axis and FoxP3 may work together for maintenance of peripheral tolerance but through nonoverlapping pathways. PD-1 is involved in rheostatic modulation of TCR signaling, preventing activity from effector $\mathrm{T}$ cells, whereas FoxP3 is sufficient for maintaining the integrity of Treg function. ${ }^{28}$ It has been suggested that PD-L1 and Treg FoxP3+ may work synergistically in tumoral microenvironments; PD-L1 may exert its inhibitory effects, at least in part by inducing Treg cell development, maximizing tumoral immune escape. ${ }^{29}$ Although increased Treg FoxP3+ infiltration and PD-L1 tumoral expression have been revealed in several malignancies, their correlation is yet undetermined. This relationship has been consistently associated with worst prognosis in breast, gastric and colorectal cancers. ${ }^{30-32}$ Our FoxP3 + lymphocyte infiltration was not impacted by tumoral PD-L1 expression ( $p>0.99)$.

Karim et al were the first to examine the relationship between the PD-1/PD-L1 axis and lymphocytic biomarkers in cervical cancer. ${ }^{17}$ The volume of infiltration by each T-cell was divided into low and high categories, determined by the 50 th percentile, and $66 \%$ of the samples exhibited a high rate of infiltration by CD8+ lymphocytes, $54 \%$ by CD4+ lymphocytes and $59 \%$ by FoxP3+ lymphocytes. The CD8+/FoxP3+ T lymphocyte ratio represented an independent positive prognostic factor for survival. 
Tumoral PD-L1 was associated with the greatest infiltration by FoxP $3+\mathrm{T}$ cells $(p=0.022)$. OS of patients with PDL1-positive tumors and a low rate of CD8+/FoxP3+ lymphocyte infiltration was significantly better than that of patients with PD-L1-negative tumors with a low CD8 +/FoxP3+ lymphocyte ratio $(p=0.033)$. In contrast, our lymphocyte analyses are not in agreement with the findings of the above authors: FoxP3+ immune infiltrates did not modify oncologic endpoints. Furthermore, PD-L1 expression in the cervical tumor specimens did not seem to affect immune infiltration degree, FoxP3 expression, nor survival outcomes.

Regarding current therapies, the results for checkpoint inhibitors in cervical neoplasia are scarce and preliminary. A Phase I trial in progress (NCT01711515) is assessing the effect of ipilimumab in patients with cervical cancer at CSs IB2-IIA (if only the para-aortic lymph nodes are compromised) or CSs IIB, IIB and IVA after chemoradiotherapy (including brachytherapy). ASCO's 2017 edition revealed the first results of Phase I/II multicohort trial (in progress) for nivolumab (CHECKMATE-358) for patients with virusassociated metastatic tumors of the cervix, vulva and vagina. Patients were not PD-L1-selected. Among the 24 treated patients, 19 (79.2\%) had cervical neoplasia. At a median follow-up of 31 weeks, the objective response rate was $20.8 \%$ - all recorded responses were from patients with cervical cancer and they were not related to PD-L1 expression, HPV status nor exposure to previous lines of therapy. ${ }^{33}$ Dr. Lheureux et al published in 2017 the results for their Phase I/II trial evaluating ipilimumab in cervical cancer. The anti-CTLA-4 therapy did not show significant single-agent activity. In addition, no increase in intratumoral CD3, CD4, CD8 and FoxP3 expression was observed before and during treatment with ipilimumab. The presence of baseline immune infiltrate was not associated with response or stable disease. ${ }^{34}$ In 2018, the Food and Drug Administration (FDA) approved pembrolizumab for recurrent or metastatic cervical cancer on or after chemotherapy whose tumors express PD-L1 based on a single cohort of KEYNOTE-158. The reported objective response rate was $14.3 \%$.

\section{Conclusion}

The lack of studies regarding cervical cancer and the immune context has led to a paucity of contemporary antineoplastic strategies that is inconsistent with the high prevalence and morbidity of this disease. Consequently, an immune checkpoint inhibitor has been approved for this scenario based on unimpressive responses, regardless of a biologic rationale uncertainty. Therefore, identification of potentially responsive patients is imperative, and thus, searching for biomarkers that predict therapeutic benefit is crucial.

Our findings must be considered with caution. Similarly to previous studies on cervical malignancy, our sample size is limited and might have impaired power to draw robust conclusions. Moreover, the controversy regarding PD-L1's predictive value has been widely discussed. IHC-based detection presents technical issues, different assays present discordant results, and it can fail to reveal the dynamic interaction of the tumoral microenvironment. Nevertheless, it remains relevant.

The results reported here reinforce that PD-L1 tumoral expression alone might be an inadequate biomarker for prediction of survival in cervical cancer. Notwithstanding, the high prevalence of FoxP3+ lymphocytic infiltrates, irrespective of PD-L1 expression, suggests that FoxP3 pathway inhibition can be a potential therapeutic approach. Furthermore, PD-L1 and Treg FoxP3+ may work synergistically and their upregulated expressions may promote tumoral evasion from immunity. Combinatorial immunotherapeutic approaches aiming PD-L1 blockage and Treg depletion might improve therapeutic efficacy. To clarify the immune escape mechanism of this neoplasm and explore effective therapies, further basic and translational researches evaluating the interactions between immune cells and cervical cancer tissue is imminent.

\section{Ethics declaration}

This study's involvement with human subjects complies with the Declaration of Helsinki.

\section{Acknowledgments}

All researchers signed a secrecy and confidentiality agreement after receiving approval by the Institutional Scientific Council and by the National Research Ethics Committee.

\section{Disclosure}

The authors report no conflicts of interest in this work.

\section{References}

1. Bray F, Ferlay J, Soerjomataram I, Siegel RL, Torre LA, Jemal A. Global cancer statistics 2018: GLOBOCAN estimates of incidence and mortality worldwide for 36 cancers in 185 countries. CA Cancer $J$ Clin. 2018;68:394-424. doi:10.3322/caac.21492

2. Instituto Nacional De Câncer José Alencar Gomes Da Silva. Estimativa da incidência e mortalidade por câncer no Brasil 2018; 2018. Available from: http://www.inca.gov.br/estimativa/2018/estima tiva-2018.pdf. Accessed September 11, 2018. 
3. Bosch FX, Lorincz A, Muñoz N, Meijer CJLM, Shah KV. The causal relation between human papillomavirus and cervical cancer. $J$ Clin Pathol. 2002;55(4):244-265. doi:10.1136/jcp.55.4.244

4. Zur Hausen H. Papillomaviruses and cancer: from basic studies to clinical application. Nat Rev Cancer. 2002;2(5):342-350. doi: $10.1038 /$ nrc 798

5. Dürst M, Gissmann L, Ikenberg H, Zur Hausen H. A papillomavirus DNA from a cervical carcinoma and its prevalence in cancer biopsy samples from different geographic regions. Proc Natl Acad Sci U S A. 1983;80(12):3812-3815.

6. Menderes G, Black J, Schwab CL, Santin AD. Immunotherapy and targeted therapy for cervical cancer: an update. Expert Rev Anticancer Ther. 2016;16(1):83-98. doi:10.1586/14737140. 2016.1121108

7. Senba M, Mori N. Mechanisms of virus immune evasion lead to development from chronic inflammation to cancer formation associated with human papillomavirus infection. Oncol Rev. 2012;6(2): e17. doi:10.4081/oncol.2012.e17

8. Tindle RW. OPINIONImmune evasion in human papillomavirusassociated cervical cancer. Nat Rev Cancer. 2002;2(1):59-64. doi: $10.1038 /$ nrc700

9. Seresini S, Origoni M, Lillo F, et al. IFN- $\gamma$ produced by human papilloma virus-18 E6-specific CD4+ T outcome cells predicts the clinical after surgery in patients with high-grade cervical lesions. J Immunol. 2007;179(10):7176-7183. doi:10.4049/jimmunol.179.10.7176

10. Pardoll DM. The blockade of immune checkpoints in cancer immunotherapy. Nat Rev Cancer. 2012;12(4):252-264. doi:10.1038/ nrc3239

11. Dong H, Strome SE, Salomao DR, et al. Tumor-associated B7-H1 promotes T-cell apoptosis: a potential mechanism of immune evasion. Nat Med. 2002;8(8):793-800. doi:10.1038/nm730

12. Enwere EK, Kornaga EN, Dean M, et al. Expression of PD-L1 and presence of CD8-positive $\mathrm{T}$ cells in pre-treatment specimens of locally advanced cervical cancer. Mod Pathol. 2017;30(4):577-586. doi:10.1038/modpathol.2016.221

13. Tanaka A, Sakaguchi S. Regulatory T cells in cancer immunotherapy. Cell Res. 2017;27(1):109-118. doi:10.1038/cr.2016.151

14. Shang B, Liu Y, Jiang S, Liu Y. Prognostic value of tumor-infiltrating FoxP3+ regulatory $\mathrm{T}$ cells in cancers: a systematic review and metaanalysis. Sci Rep. 2015;5(1):15179. doi:10.1038/srep15179

15. McShane LM, Altman DG, Sauerbrei W, Taube SE, Gion M, Clark GM. Reporting recommendations for tumor marker prognostic studies. J Clin Oncol. 2005;23(36):9067-9072. doi:10.1200/ JCO.2004.01.0454

16. Heeren AM, Punt S, Bleeker MC, et al. Prognostic effect of different PD-L1 expression patterns in squamous cell carcinoma and adenocarcinoma of the cervix. Mod Pathol. 2016;29(7):753-763. doi:10.1038/modpathol.2016.64

17. Karim R, Jordanova ES, Piersma SJ, et al. Tumor-expressed B7-H1 and B7-DC in relation to PD-1+ T-cell infiltration and survival of patients with cervical carcinoma. Clin Cancer Res. 2009;15 (20):6341-6347. doi:10.1158/1078-0432.CCR-09-1652

18. Huang C, Zhou L, Chang X, Pang X, Zhang H, Zhang S. B7-H3, B7H4, Foxp3 and IL-2 expression in cervical cancer: associations with patient outcome and clinical significance. Oncol Rep. 2016;35 (4):2183-2190. doi:10.3892/or.2016.4607

19. Luo Q, Zhang S, Wei H, Pang X, Zhang H. Roles of Foxp3 in the occurrence and development of cervical cancer. Int J Clin Exp Pathol. 2015;8(8):8717-8730.
20. Triulzi T, Tagliabue E, Balsari A, Casalini P. FOXP3 expression in tumor cells and implications for cancer progression. J Cell Physiol. 2013;228(1):30-35. doi:10.1002/jcp.24125

21. Wang X, Teng F, Kong L, Yu J. PD-L1 expression in human cancers and its association with clinical outcomes. Onco Targets Ther. 2016;9:5023-5039. doi:10.2147/OTT.S105862

22. Santin AD, Bellone S, Palmieri M, et al. Induction of tumor-specific cytotoxicity in tumor infiltrating lymphocytes by HPV 16 and HPV 18 E7-pulsed autologous dendritic cells in patients with cancer of the uterine cervix. Gynecol Oncol. 2003;89(2):271-280. doi:10.1016/ S0090-8258(03)00083-0

23. Mabuchi S, Matsumoto Y, Kawano M, et al. Uterine cervical cancer displaying tumor-related leukocytosis: a distinct clinical entity with radioresistant feature. J Natl Cancer Inst. 2014;106(7):dju147dju147. doi:10.1093/jnci/dju147

24. Hilders CG, Ras L, van Eendenburg JD, Nooyen Y, Fleuren GJ. Isolation and characterization of tumor-infiltrating lymphocytes from cervical carcinoma. Int J Cancer. 1994;57(6):805-813.

25. Evans EM, Man S, Evans AS, Borysiewicz LK. Infiltration of cervical cancer tissue with human papillomavirus-specific cytotoxic Tlymphocytes. Cancer Res. 1997;57(14):2943-2950.

26. Jordanova ES, Gorter A, Ayachi O, et al. Human leukocyte antigen class I, MHC class I chain-related molecule A, and CD8+/regulatory T-cell ratio: which variable determines survival of cervical cancer patients? Clin Cancer Res. 2008;14(7):2028-2035. doi:10.1158/ 1078-0432.CCR-07-4554

27. Matsumoto Y, Mabuchi S, Kozasa K, et al. The significance of tumorassociated neutrophil density in uterine cervical cancer treated with definitive radiotherapy. Gynecol Oncol. 2017;145(3):469-475. doi:10.1016/j.ygyno.2017.02.009

28. Zhang B, Chikuma S, Hori S, Fagarasan S, Honjo T. Nonoverlapping roles of PD-1 and FoxP3 in maintaining immune tolerance in a novel autoimmune pancreatitis mouse model. Proc Natl Acad Sci. 2016;113 (30):8490-8495. doi:10.1073/pnas.1608873113

29. Francisco LM, Salinas VH, Brown KE, et al. PD-L1 regulates the development, maintenance, and function of induced regulatory $\mathrm{T}$ cells. $J$ Exp Med. 2009;206(13):3015-3029. doi:10.1084/jem. 20090847

30. Li Z, Dong P, Ren M, et al. PD-L1 expression is associated with tumor FOXP3 $^{+}$regulatory T-cell infiltration of breast cancer and poor prognosis of patient. $J$ Cancer. 2016;7(7):784-793. doi:10.7150/jca. 14549

31. Hou J, Yu Z, Xiang R, et al. Correlation between infiltration of FOXP3+ regulatory $\mathrm{T}$ cells and expression of $\mathrm{B} 7-\mathrm{H} 1$ in the tumor tissues of gastric cancer. Exp Mol Pathol. 2014;96(3):284-291. doi:10.1016/j.yexmp.2014.03.005

32. Zhao L, Li C, Zhang R, et al. B7-H1 and B7-H4 expression in colorectal carcinoma: correlation with tumor FOXP3+ regulatory Tcell infiltration. Acta Histochem. 2014;116(7):1163-1168. doi:10.1016/j.acthis.2014.06.003

33. Hollebecque A, Meyer T, Moore KN, et al. An open-label, multicohort, phase I/II study of nivolumab in patients with virusassociated tumors (CheckMate 358): efficacy and safety in recurrent or metastatic (R/M) cervical, vaginal, and vulvar cancers. J Clin Oncol. 2017;35(15 suppl):5504. doi:10.1200/ JCO.2017.35.15_suppl.5504

34. Lheureux S, Butler MO, Clarke B, et al. Association of ipilimumab with safety and antitumor activity in women with metastatic or recurrent human papillomavirus-related cervical carcinoma. JAMA Oncol. 2018;4(7):e173776. doi:10.1001/jamaoncol.2017.3776 


\section{Publish your work in this journal}

Cancer Management and Research is an international, peer-reviewed open access journal focusing on cancer research and the optimal use of preventative and integrated treatment interventions to achieve improved outcomes, enhanced survival and quality of life for the cancer patient.
The manuscript management system is completely online and includes a very quick and fair peer-review system, which is all easy to use. Visit http://www.dovepress.com/testimonials.php to read real quotes from published authors.

Submit your manuscript here: https://www.dovepress.com/cancer-management-and-research-journal 